\title{
Effect of Reconstruction Algorithms on Myocardial Blood Flow Measurement with ${ }^{13} \mathrm{~N}-\mathrm{Ammonia} \mathrm{PET}$
}

Grace P. Chen ${ }^{1}$, Kelley R. Branch ${ }^{1}$, Adam M. Alessio², Pam Pham² ${ }^{2}$ Ramin Tabibiazar ${ }^{3}$, Paul Kinahan ${ }^{2}$, and James H. Caldwell ${ }^{1,2}$

${ }^{1}$ Division of Cardiology, Department of Medicine, University of Washington, Seattle, Washington; ${ }^{2}$ Department of Radiology, University of Washington, Seattle, Washington; and ${ }^{3}$ Division of Cardiology, Department of Medicine, University of California, Los Angeles, California

Filtered backprojection (FBP) is the traditional method for ${ }^{13} \mathrm{~N}-$ $\mathrm{NH}_{3}$ PET studies. Ordered-subsets expectation maximization (OSEM) is popular for PET studies because of better noise properties. Scant data exist on the effect of reconstruction algorithms on quantitative myocardial blood flow (MBF) estimation. Methods: Twenty patients underwent dynamic acquisition rest/stress ${ }^{13} \mathrm{~N}-\mathrm{NH}_{3}$ studies. In Part 1, 19 rest/stress image pairs were reconstructed by FBP (10-mm Hanning filter) and by OSEM with 28 subsets and 2 (OSEM2), 6 (OSEM6), or 8 iterations (OSEM8), and a 10-mm postreconstruction smoothing gaussian filter. In Part 2, 9 image pairs were reconstructed by FBP (10-mm Hanning filter) and by OSEM with 28 subsets, 8 iterations, and a gaussian 5-, 10-, or 15-mm postreconstruction smoothing filter. Average MBF ( $\mathrm{mL} / \mathrm{min} / \mathrm{mL}$ of myocardium) was calculated using a 3-compartment model. Results: Part 1: For rest MBF, the correlations between FBP and each of the OSEM algorithms were $r^{2}=0.71,0.73$, and 0.77 , respectively. MBF by OSEM6 $(0.98 \pm$ 0.48 [mean \pm SD]) and OSEM8 $(0.96 \pm 0.46)$ was not significantly different from FBP $(1.02 \pm 0.39)$, but OSEM2 $(0.80 \pm 0.37)$ was significantly lower $(P<0.0003)$. With stress, the correlations were high between FBP and OSEM6 and OSEM8 $\left(r^{2}=0.85\right.$ and 0.90$)$, and MBF by OSEM 6 and OSEM 8 was not significantly different from FBP. Part 2: Resting MBF correlated well between FBP and all OSEM smoothing filters $\left(r^{2}=0.82,0.85\right.$, and 0.88). Rest MBF using postsmoothing 5- or $10-\mathrm{mm}$ filters was not different from FBP but was significantly lower with the 15-mm filter $(P<0.05)$. With stress, the correlations were good between FBP and OSEM regardless of smoothing $\left(r^{2}=0.76,0.77\right.$, and 0.79$)$. However, MBF with postsmoothing 10- and 15-mm filters was significantly lower than by FBP $(P<0.05)$. Conclusion: Reconstruction algorithms significantly affect the estimation of quantitative blood flow data and should not be assumed to be interchangeable. Although aggressive smoothing may produce visually appealing images with reduced noise levels, it may cause an underestimation of absolute quantitative MBF. In selecting a reconstruction algorithm, an optimal balance between noise properties and diagnostic accuracy must be emphasized.

Received Nov. 17, 2006; revision accepted May 7, 2007.

For correspondence or reprints contact: James H. Caldwell, MD, Nuclear Medicine, Box 356113, University of Washington, Seattle, WA 98195.

E-mail: jcald@u.washington.edu

COPYRIGHT @ 2007 by the Society of Nuclear Medicine, Inc.
Key Words: PET; reconstruction algorithms; myocardial blood flow

J Nucl Med 2007; 48:1259-1265

DOI: 10.2967/jnumed.106.038232

$\mathbf{T}$ he temporal acquisition capacity of PET allows for quantitative assessment of myocardial blood flow (MBF), which is not possible currently with SPECT. ${ }^{13} \mathrm{~N}_{-} \mathrm{NH}_{3}$ is frequently used in clinical cardiac PET. Tracer kinetic models have been developed to describe the exchange of $\mathrm{NH}_{3}$ between the vascular space and myocardial tissue to quantify MBF $(1,2)$. MBF as determined by cardiac PET with ${ }^{13} \mathrm{~N}^{-\mathrm{NH}_{3}}$ has been validated against blood flow measurements by microspheres in animal experiments (3), by PET with ${ }^{15} \mathrm{O}$-water (4), and by argon inert gas in humans (5).

Reconstruction is an essential step in the processing of PET images. Commonly used reconstruction algorithms for PET follow 2 main categories: (i) analytic, such as filtered backprojection (FBP); and (ii) iterative, such as orderedsubsets expectation maximization (OSEM) (6). FBP is the traditional method for ${ }^{13} \mathrm{~N}-\mathrm{NH}_{3}$ PET (7). It provides a fast and direct solution and is well suited for clinical use. However, OSEM has gained popularity recently because of the noise reduction properties in regions of low uptake (8). However, it is known that image resolution and noise increase with an increasing number of iterations $(9,10)$, but the relative trade-offs for both visualization and measurement tasks are unclear.

Limited data exist on the effects of reconstruction algorithms on PET data in cardiac studies $(9,11)$. Moreover, the data in cardiac studies pertain only to ${ }^{18}$ F-FDG PET studies. Lubberink et al. compared FBP and OSEM (16 subsets, 6 iterations) in cardiac ${ }^{18} \mathrm{~F}-\mathrm{FDG}$ PET studies of 8 patients (11). Scatter plots of the ${ }^{18}$ F-FDG influx rate constants showed linear correlation between values obtained with FBP and with OSEM. Boellaard et al. compared 5 reconstruction algorithms in 5 cardiac ${ }^{18}$ F-FDG PET studies (9). 
The reconstruction algorithms included one FBP algorithm and several OSEM algorithms with varying iterations and subsets and varying amounts of smoothing. The authors found significant changes to the metabolic rate of glucose depending on the reconstruction algorithm and on the use of smoothing. The effects of reconstruction algorithms on quantitative MBF have not been tested. This study was undertaken because we observed a marked difference in the shape of the heart and in regional abnormalities when we compared images reconstructed using the equipment vendor's default OSEM parameters (2 iterations, 28 subsets) with images reconstructed using FBP (Fig. 1). This raised questions about the effects of iterative reconstruction on qualitative and quantitative MBF. The goals of the current study were:

(a) To define the effects of the number of OSEM iterations on absolute rest and vasodilator stress MBF and coronary flow reserve (CFR) estimated by ${ }^{13} \mathrm{~N}-$ $\mathrm{NH}_{3} \mathrm{PET}$;

(b) To assess the effects of smoothing in OSEM on the estimation of absolute rest and vasodilator stress MBF and CFR by ${ }^{13} \mathrm{~N}-\mathrm{NH}_{3}$ PET; and

(c) To compare the OSEM results with those obtained by the conventional FBP reconstruction algorithm.

\section{MATERIALS AND METHODS}

\section{Study Population}

The study population consisted of 20 patients (17 men, 3 women; mean age $\pm \mathrm{SD}, 60 \pm 14 \mathrm{y}$; age range, 13-72 y) who underwent cardiac ${ }^{13} \mathrm{~N}^{-\mathrm{NH}_{3}}$ studies for evaluation of myocardial ischemia. All patients were studied at rest and during hyperemia induced by intravenous infusion of the vasodilator dipyridamole at $0.57 \mathrm{mg} / \mathrm{kg}$ over $4 \mathrm{~min}$. Continuous blood pressure and electrocardiographic monitoring was performed during the stress test by a cardiologist or a cardiology nurse practitioner. There were no complications in executing the study protocol.

\section{PET}

Images were acquired in 2-dimensional mode on an Advance PET scanner (GE Healthcare). After the 1-min scout scan for patient positioning, a 20-min transmission scan for the selected bed position (axial field of view, $16 \mathrm{~cm}$ ) was obtained for attenuation correction. For the rest scan, $858 \pm 137 \mathrm{MBq}(23.2 \pm$ $3.7 \mathrm{mCi}[$ mean $\pm \mathrm{SD}])$ of ${ }^{13} \mathrm{~N}_{-\mathrm{NH}_{3}}$ was injected as a bolus $(<3 \mathrm{~s})$ with a 4-min dynamic image acquisition $(15 \times 2 \mathrm{~s}, 6 \times 5 \mathrm{~s}, 6 \times 10 \mathrm{~s}$, $8 \times 15 \mathrm{~s}$ ). Thereafter, a 15 -min gated acquisition was performed using 16 bins and 30-ms intervals. After an additional $20 \mathrm{~min}$ for decay of ${ }^{13} \mathrm{~N}$ activity, dipyridamole infusion was given over $4 \mathrm{~min}$ while a 4-min stress transmission scan was being performed for attenuation correction of the stress image data. A bolus injection of $1,081 \pm 203 \mathrm{MBq}(29.2 \pm 5.5 \mathrm{mCi}[$ mean $\pm \mathrm{SD}])$ of ${ }^{13} \mathrm{~N}-\mathrm{NH}_{3}$ was then given; this was followed immediately by a 4-min dynamic acquisition and a 15-min gated acquisition. The gated images were used for qualitative assessment of ischemia. The dynamic images were reconstructed for quantification of MBF.

\section{PET Image Reconstruction and Processing}

For Part 1 of the study, the dynamic rest and stress data were corrected for attenuation, scattered and random coicidences, detector efficiency variations, and dead time and reconstructed by FBP and by various OSEM algorithms into $128 \times 128 \times 35$ image volumes. For FBP, a 10-mm Hanning filter was used, which is the standard method for our clinic. For OSEM, the images were reconstructed using 28 subsets and either 6 iterations (OSEM6) or 8 iterations (OSEM8). The OSEM images were postreconstruction smoothed with a $10-\mathrm{mm}$ gaussian filter. In addition to these reconstruction
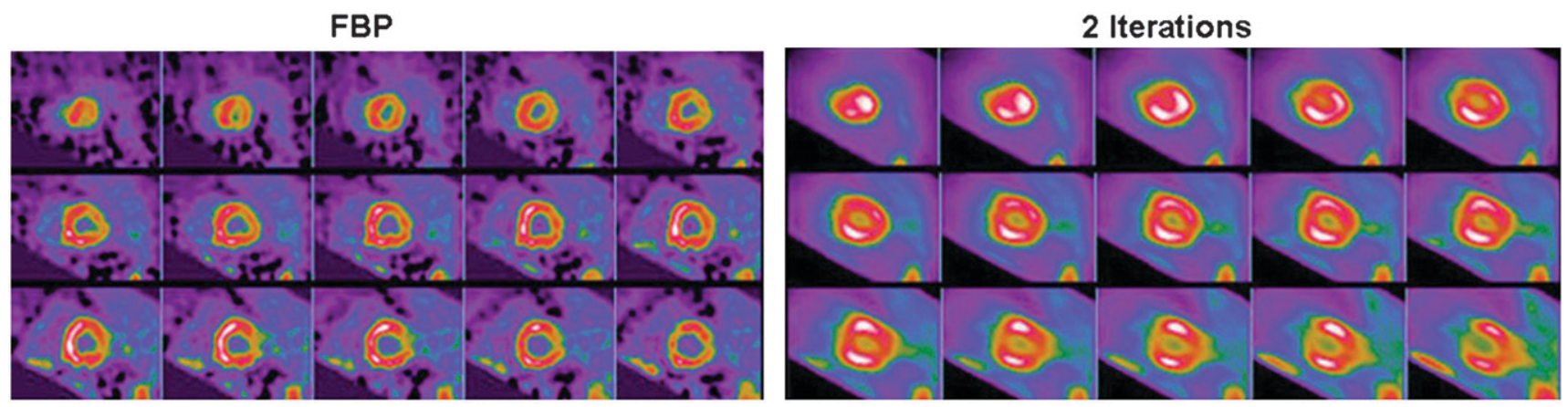

6 Iterations

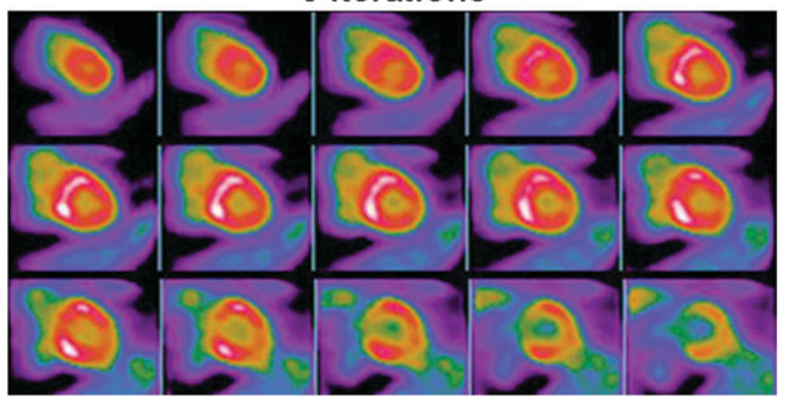

FIGURE 1. Short-axis images reconstructed using FBP and OSEM with 28 subsets and either 2 or 6 iterations. Of interest is anterior-septal defect on 2-iteration image set that is not present on FBP or 6-iteration OSEM images. 
methods, the rest images were also reconstructed by OSEM with 2 iterations (OSEM2). Stress images were not reconstructed with OSEM2 because of the poor results with the rest images.

For Part 2 of the study, the dynamic rest and stress data were reconstructed by FBP and by OSEM with 28 subsets and 8 iterations. The FBP algorithm again used a 10-mm Hanning filter. For OSEM, the images were postsmoothed with a gaussian 5-, 10-, or 15-mm filter. For the purpose of the Discussion, these will be denoted as OSEMps5, OSEMps10, and OSEMps15, respectively.

The reconstructed images were then reoriented to form 30 shortaxis images with 35 time bins each and a slice thickness of $4.25 \mathrm{~mm}$. A small circular region of interest (ROI), 3-pixel diameter, was placed in the center of the left atrium on the 3 short-axis slices best defining the left atrial tracer bolus for calculation of the arterial input function. Data from these 3 ROIs were averaged to form a single left atrial time-activity curve. A semiautomatic computer algorithm determined the circumferential myocardial midline of activity on 12-16 LV short-axis slices (depending on the size of the heart, but the same for both rest and stress studies in the same patient) and generated a 2-pixel-wide ROI centered on the midline. These ROIs were then used to create an average myocardial ROI, and a timeactivity curve was generated. The average $\mathrm{MBF}(\mathrm{mL} / \mathrm{min} / \mathrm{g}$ of myocardium) was measured from the average left atrial and myocardial time-activity curves using the 3-compartment model developed at the University of Michigan (3). The model was implemented in software (gentex2) developed in the University of Washington National Simulation Research facility (James Bassingthwaighte) but used midmyocardial regions (2-pixel wide on either side of the midline) instead of endocardial regions of the Michigan model. This might give slightly different absolute values of MBF in an individual patient compared with the value determined by the exact Michigan model, but this is not important for the purpose of the comparisons in our study. Using the myocardial midline as the center for the ROIs, rather than an endocardial one, should reduce the difference between FBP and OSEM values - as visually, much of the effect of OSEM appears to be at the endocardial and epicardial margins in the dynamic image sets. Processing tools and the graphical user interface were developed in our laboratory (12). CFR was calculated by dividing stress MBF by rest MBF.

\section{Intraobserver and Interobserver Variability}

To examine potential effects of intra- and interobserver variability on our results, 5 patients ( 2 healthy, 2 with ischemia by visual assessment of the PET images, and the patient with the greatest difference between FBP and OSEM2 values) were selected. New left atrial and myocardial ROIs were defined by 1 author on 2 separate occasions over a 2 -wk period without knowledge of which image planes were selected at the time of the original analysis $>6$ mo earlier or the previous analysis. Rest and stress FBP images sets were analyzed for the 5 selected patients and a random selection of the 3 OSEM protocols. All patients had 2 OSEM protocols-for example, OSEM2 and OSEM8, examined at rest and an OSEM8 stress protocol. Interobserver variability was done by comparing the results from the first analysis with the average result of the intraobserver measurements. Differences were calculated as the absolute difference.

\section{Statistical Analysis}

Values are expressed as mean \pm SD. Regression analysis was used to describe the correlation between results from FBP and results from the various OSEM algorithms. Group comparisons between results obtained from the FBP and from each of the OSEM algorithms were performed using a paired $t$ test. Analysis was done for average resting $\mathrm{MBF}$, average stress $\mathrm{MBF}$, and CFR.

\section{RESULTS}

\section{Part 1: OSEM Versus FBP}

All values for $\mathrm{MBF}$ are expressed as $\mathrm{mL} / \mathrm{min} / \mathrm{mL}$ of myocardium. For rest MBF, the correlations were good between FBP and OSEM2 $\left(r^{2}=0.71\right)$, FBP and OSEM6 $\left(r^{2}=0.73\right)$, and FBP and OSEM8 $\left(r^{2}=0.77\right)$. MBF by OSEM6 $(0.98 \pm 0.48)$ and OSEM8 $(0.96 \pm 0.46)$ were not significantly different from FBP $(1.02 \pm 0.39)(P>0.05)$, but MBF by OSEM2 $(0.80 \pm 0.37)$ was significantly lower than MBF by FBP $(P<0.0003)$ (Fig. 2).

For stress MBF, the correlations were good between FBP and OSEM6 $\left(r^{2}=0.85\right)$ and FBP and OSEM8 $\left(r^{2}=0.90\right)$. MBF by OSEM6 $(2.44 \pm 1.24)$ and OSEM8 $(2.44 \pm 1.16)$

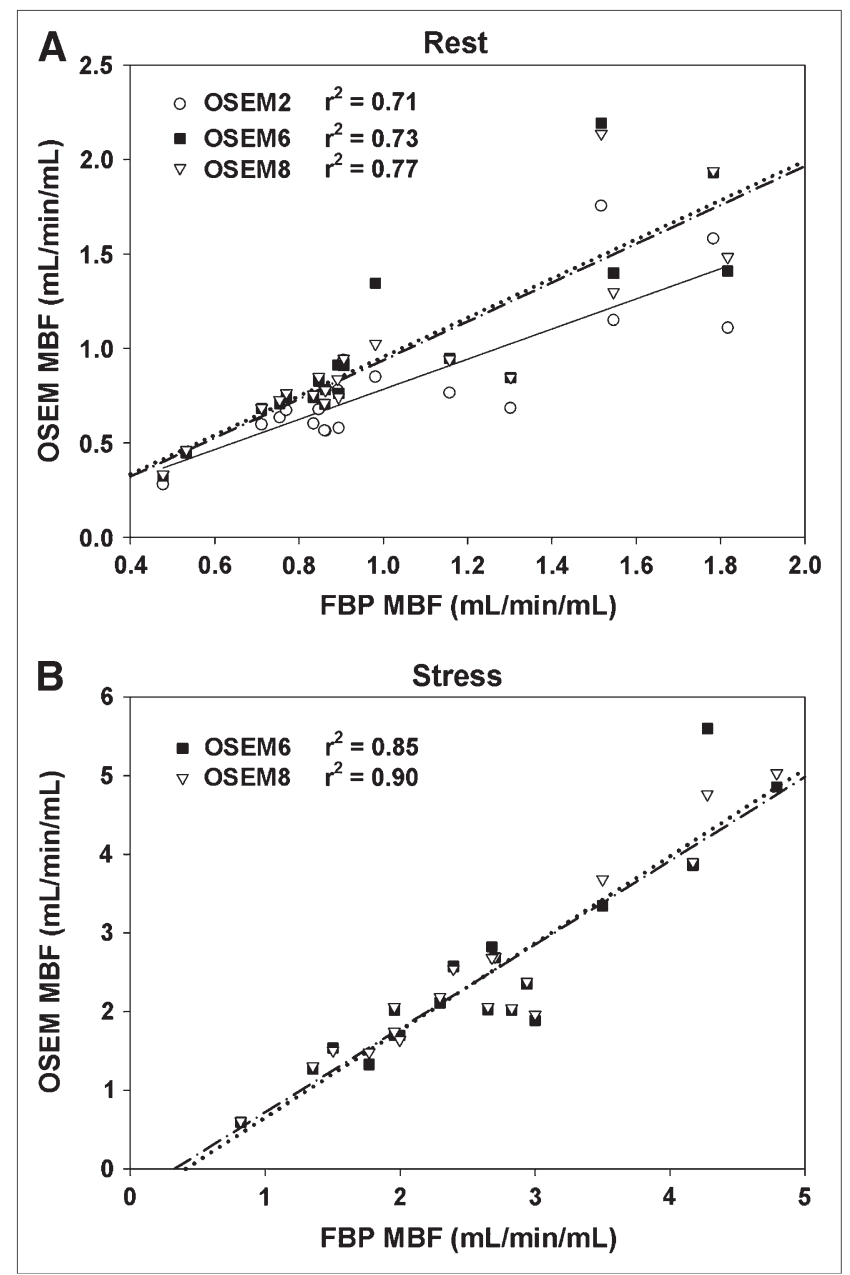

FIGURE 2. (A) Comparison of rest MBF by each OSEM iteration (OSEM2, OSEM6, and OSEM8) with MBF by FBP reconstruction. Correlations were highly significant for each method as shown by $r^{2}$ values. However, at higher flows, OSEM2 consistently underestimated MBF compared with OSEM6 and OSEM8. (B) The same comparisons are made for stress MBF for OSEM6 and OSEM8, with even higher correlations and less variability. 
was not significantly different from FBP $(2.61 \pm 1.03)(P$ $>0.05$ ) (Fig. 3).

Similarly, for CFR the correlations were good between FBP and OSEM6 $\left(r^{2}=0.77\right)$ and FBP and OSEM8 $\left(r^{2}=\right.$ 0.80). CFR by OSEM6 (2.56 \pm 0.77$)$ and OSEM8 $(2.65 \pm$ $0.92)$ was not significantly different from FBP (2.71 \pm 0.98) $(P>0.05)$.

\section{Part 2: Effects of Smoothing}

In Part 2 of the study, resting MBF correlated well between FBP and OSEMps5 $\left(r^{2}=0.82\right)$, FBP and OSEMps 10 $\left(r^{2}=0.85\right)$, and FBP and OSEMps15 $\left(r^{2}=0.88\right)$. MBF by OSEMps5 (0.94 \pm 0.49$)$ and OSEMps10 (0.88 \pm 0.44$)$ was not significantly different from FBP $(1.00 \pm 0.39)(P>$ $0.05)$, but MBF by OSEMps $15(0.81 \pm 0.39)$ was significantly lower than MBF by FBP $(P<0.003)$.

For stress MBF, the correlations were good between FBP and OSEMps5 $\left(r^{2}=0.76\right)$, FBP and OSEMps10 $\left(r^{2}=\right.$ $0.77)$, and FBP and OSEMps15 $\left(r^{2}=0.79\right)$. MBF by OSEMps5 $(1.92 \pm 0.54)$ was not significantly different from FBP $(2.11 \pm 0.72)(P>0.05)$, but MBF by OSEMps 10 $(1.80 \pm 0.54)$ and OSEMps15 (1.65 \pm 0.49$)$ was significantly lower than MBF by FBP $(P<0.03$ and $P<0.005$, respectively) (Fig. 4).

Finally, there was excellent correlation in CFR between FBP and OSEMps5 $\left(r^{2}=0.90\right)$, FBP and OSEMps10 $\left(r^{2}=\right.$ $0.91)$, and FBP and OSEMps15 $\left(r^{2}=0.89\right)$. CFR by OSEMps5 (2.26 \pm 0.72), OSEMps10 (2.23 \pm 0.74$)$, and OSEMps15 (2.22 \pm 0.76$)$ was not significantly different from $\operatorname{FBP}(2.33 \pm 1.07)(P>0.05)$.

\section{Intraobserver and Interobserver Variability}

There was minimal intraobserver variability for FBP or OSEM reconstructed images, as shown in Table 1. Interobserver differences were also small with the exception of the rest OSEM comparison, in which there was a small, but

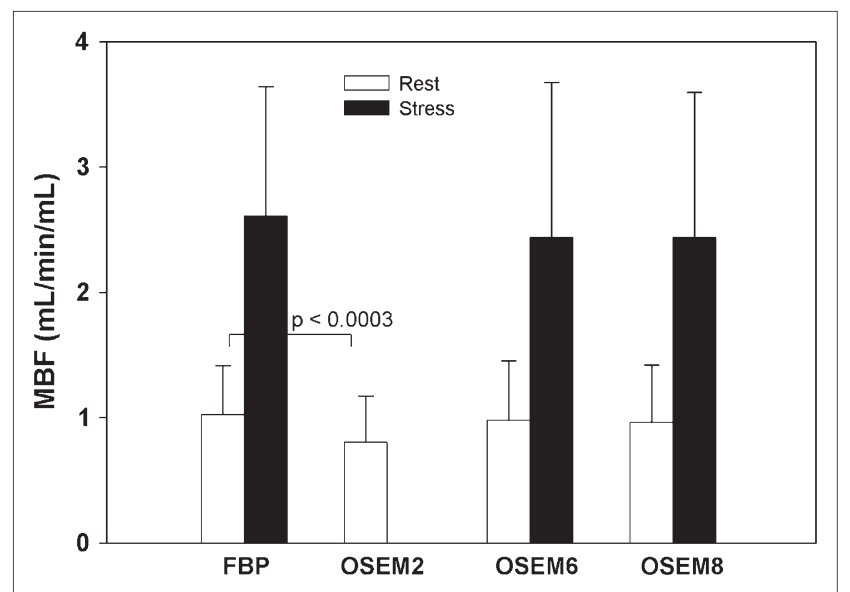

FIGURE 3. Mean \pm SD of rest and stress MBF by each of reconstruction methods is shown. There is a slight, but statistically insignificant, tendency for MBF to be lower with OSEM6 and OSEM8 iterations. For OSEM2 at rest, MBF was significantly lower.

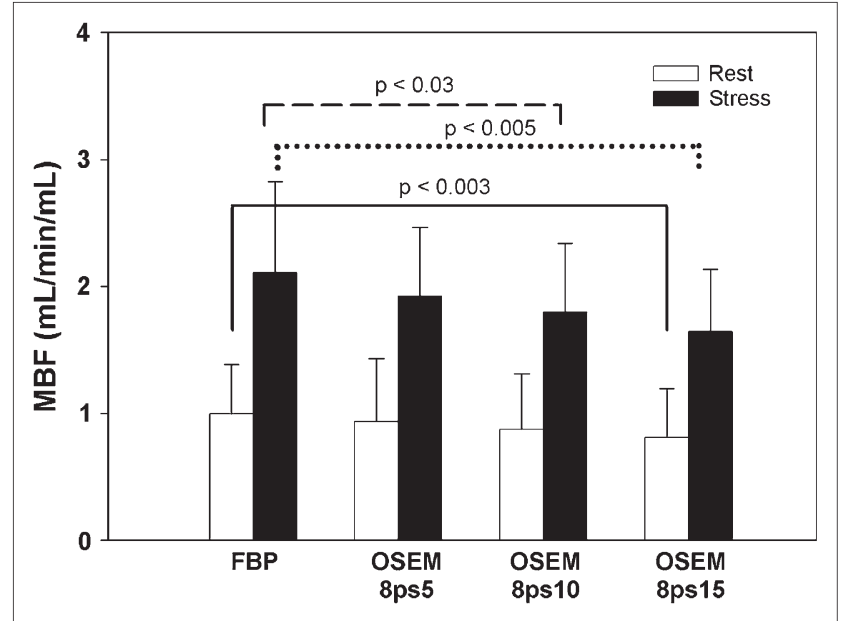

FIGURE 4. Effect of increasing filter function with OSEM reconstruction at a constant number of iterations $(n=8)$ is to decrease mean MBF compared with FBP. This was statistically significant only at a filter function of 15 (ps15) at rest whereas, for stress MBF, this occurred at filter functions of 10 (ps10) and 15 (ps15).

statistically significant, difference. This difference (mean, $0.08 \mathrm{~mL} / \mathrm{min} / \mathrm{mL}$ ) was driven by a single OSEM2 and a single OSEM8 comparison because of differences in placement of the left atrial ROI.

\section{DISCUSSION}

Our study systematically evaluates the effect of changing the number of iterations and the filter function on the measurement of absolute MBF. For rest MBF, OSEM2, OSEM6, and OSEM8 iterations correlate well with FBP, but OSEM2 causes significant underestimation of MBF. For stress MBF, both OSEM6 and OSEM8 correlate well with the standard FBP method. Aggressive smoothing with a 15-mm filter causes an underestimation of both rest MBF and stress MBF compared with FBP. Smoothing with a 10-mm filter causes an underestimation of stress MBF compared with FBP. CFR correlated well among all algorithms, and the calculated values were not significantly different among the algorithms.

The values we report for rest and stress MBF using FBP reconstruction methods fall well within the range reported by others for rest and stress studies in a population being evaluated for ischemic heart disease $(4,5,13)$. Our CFR values by FPB are lower than those reported in middle-aged volunteers, but this would be expected as we evaluated only individuals with suspected or known coronary disease (14). Thus, our MBF and CFR values by FBP serve as a good reference against which the OSEM method can be compared.

Reconstruction of dynamic PET studies had conventionally been performed using FBP. This algorithm provides a fast, direct solution. Iterative methods, on the other hand, offer the potential for improved noise statistics. Previously, the long computation time with iterative reconstructive algorithms was sometimes prohibitive. Advances in computer processor speed and the hardware implementation of 
TABLE 1

Intraobserver and Interobserver Comparisons

\begin{tabular}{|c|c|c|c|c|c|c|}
\hline \multirow[b]{2}{*}{ Intraobserver ${ }^{*}$} & \multicolumn{3}{|c|}{ FBP (mean \pm SD) } & \multicolumn{3}{|c|}{ OSEM (mean \pm SD) } \\
\hline & Observer 1-a & Observer 1-b & Difference $^{\dagger}$ & Observer 1-a & Observer 1-b & Difference $^{\dagger}$ \\
\hline Rest & $0.80 \pm 0.10$ & $0.81 \pm 0.10$ & $0.04 \pm 0.03$ & $0.68 \pm 0.10$ & $0.69 \pm 0.11$ & $0.02 \pm 0.02$ \\
\hline \multirow[t]{2}{*}{ Stress } & $3.13 \pm 1.07$ & $3.14 \pm 1.09$ & $0.12 \pm 0.16$ & $2.78 \pm 1.40$ & $2.74 \pm 1.50$ & $0.13 \pm 0.13$ \\
\hline & \multicolumn{3}{|c|}{ FBP (mean $\pm \mathrm{SD})$} & \multicolumn{3}{|c|}{ OSEM (mean \pm SD) } \\
\hline Interobserver & Observer 1 & Observer 2 & Difference $^{\dagger}$ & Observer 1 & Observer 2 & Difference $^{\dagger}$ \\
\hline Rest & $0.80 \pm 0.09$ & $0.82 \pm 0.08$ & $0.03 \pm 0.02$ & $0.74 \pm 0.12$ & $0.68 \pm 0.11^{\ddagger}$ & $0.08 \pm 0.02$ \\
\hline Stress & $3.01 \pm 1.06$ & $3.14 \pm 1.07$ & $0.35 \pm 0.29$ & $2.83 \pm 1.26$ & $2.77 \pm 1.43$ & $0.15 \pm 0.12$ \\
\hline \multicolumn{7}{|c|}{$\begin{array}{l}{ }^{*} \text { Number of comparisons for FBP rest and stress }=5 \text { and } 8 \text { for OSEM (mixture of OSEM2, OSEM6, and OSEM8) and is the same for bot } \\
\text { intraobserver and interobserver comparisons. No statistical comparisons were made between absolute MBF values for FBP and OSEN } \\
\text { because of the mixture of OSEM iterations. } \\
{ }^{\dagger} \text { Difference = mean of the absolute difference between comparisons. } \\
{ }^{\ddagger} P=0.02 \text {, observer } 1 \text { vs. observer } 2 \text {. }\end{array}$} \\
\hline
\end{tabular}

algorithms with some vendors have certainly reduced OSEM computation limitations. Computation time also varies with the number of iterations, and the manufacturers' factory setting often uses low numbers of iterations such as OSEM2. This further cuts down on computation time but at an unknown expense of accuracy. Nevertheless, time consideration might be significant in a busy clinic. For example, reconstruction of the dynamic datasets used in this study and on the hardware described in the Materials and Methods required $4.7 \mathrm{~min}$ for FBP, $14 \mathrm{~min}$ for OSEM2, $35 \mathrm{~min}$ for OSEM6, and $49 \mathrm{~min}$ for OSEM8. When reconstructing similar image sets on a Discovery STE PET/CT system (GE Healthcare), the overall time is reduced by $30 \%$ but the relative differences remain. Because there is little difference in absolute MBF values between OSEM6 and OSEM8 and FBP and considerable time savings of OSEM6 over OSEM8, the former would seem to be a reasonable compromise if one chooses (is forced to) to use iterative reconstruction.

The observed variations in blood flow estimates with different reconstruction algorithms are the result of variations in quantitative accuracy and precision in each frame of the dynamic volume. Reconstruction algorithms and the parameters for these algorithms result in images with a tradeoff between accuracy and precision — or in other words, bias and variance. Often it is assumed that we prefer the most accurate images (with the best resolution and the fewest partial-volume errors) to find accurate tissue concentrations. Unfortunately, in PET these images also have very low precision (high variance and noise), leading to noisy estimates at each frame, which can confound the modeling process.

To our knowledge, the current study is the first to investigate the effects of reconstruction algorithms in cardiac ${ }^{13} \mathrm{~N}-\mathrm{NH}_{3}$ PET studies. We have demonstrated that, in quantifying $\mathrm{MBF}$, different reconstruction algorithms and different degrees of smoothing significantly affect the data. This type of investigation is particularly pertinent with the recent interest in iterative reconstruction. In fact, some ven- dors no longer offer an analytic (FBP) reconstruction with their latest models. The present study serves as a reminder that image quality obtained using low numbers of iterations may compromise data accuracy.

One major aim of the present study was to assess the effects of reconstruction algorithms on quantitative MBF, but not to prescribe an ideal algorithm. Another major aim of the study focused on the use of smoothing, which is a factor that has not been extensively investigated in the past. Using aggressive smoothing parameters in OSEM reconstructed images, we obtained significantly lower values of MBF than with FBP. Once again, caution must be used in trading image quality for data accuracy.

Finally, the algorithm used affects not only quantitative data, as we have shown, but also would affect qualitative interpretation, as shown in Figure 5 for normalized polar maps of $\mathrm{NH}_{3}$ activity (cpm/voxel).

Myocardial perfusion and CFR in the present study were not determined independently of PET by another reference method because no other noninvasive method is available to quantify MBF. The radioactive microsphere method, as the gold standard for measurements of MBF, requires postmortem tissue samples and can be used only in animals (15). Therefore, the FBP algorithm was used as the conventional reference method against which other algorithms were compared. Although this choice may be subject to confounders, one should keep in mind that FBP was the reconstruction method used in most of the early articles that first developed and validated absolute MBF quantification by PET. Therefore, FBP, albeit imperfect, is likely the best reference method for this kind of comparison.

A comprehensive evaluation of the effects of increasing number of iterations should ideally include more than OSEM2, OSEM6, and OSEM8. One can potentially devise a study using OSEM2, OSEM4, OSEM6, OSEM8, OSEM10, and so on. However, beyond 10 iterations, the incremental change is small. The amount of computing power and 
FIGURE 5. Polar plots of $\mathrm{NH}_{3}$ activity (cpm/voxel) with different reconstruction methods for a single patient. Activity in each of 128 myocardial ROls (8 sectors as shown by numbers on images and 16 slices) has been normalized to the maximal $\mathrm{ROI}$ in the respective plot and shown as an inverted gray scale 9 (shown in color bar). Location of maximal count region varies considerably between FBP and OSEM plots, particularly comparing FBP and OSEM2.

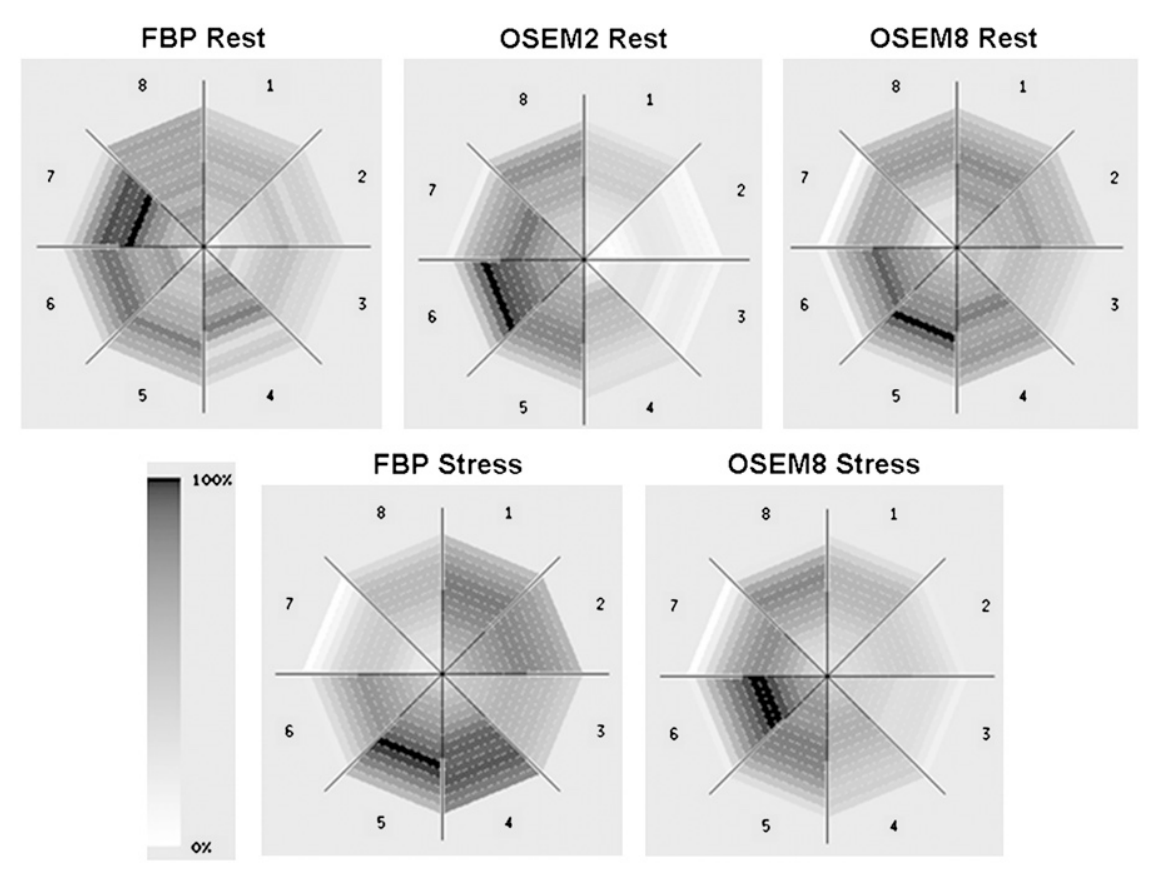

processing time required for such a full evaluation was not available to apply to the full dataset. This type of analysis was nonetheless done on 1 subject with the OSEM MBF values asymptotically approaching FBP.

As stated earlier, the implementation of the OSEM algorithm differs from manufacturer to manufacturer. GE Healthcare hardware and software were used in this study. The applicability of the findings in this study to PET images from other vendors is unknown. However, the purpose of the study was not to find the exact replacement algorithm for FBP in all clinical settings and with all manufacturers, but to answer the fundamental question of how a reconstruction algorithm affects MBF quantification. As such, the finding of a significant influence of reconstruction on MBF quantification should alert the reader not to interchange algorithms without first testing the validity of such a substitution.

Finally, we have not attempted to determine the effect of increasing the number of OSEM iterations or the smoothing filter strength on qualitative image interpretation. We believe this is an important aspect of comparing the impact of using OSEM with cardiac ${ }^{13} \mathrm{~N}^{-N_{3}}$ PET studies. This would, however, require a much larger population-that includes a wide range of disease severity plus a healthy cohort - than is available at this site.

\section{CONCLUSION}

Reconstruction algorithms significantly affect quantitative blood flow data and should not be assumed to be interchangeable. When substituting an OSEM algorithm for FBP in reconstructing cardiac $\mathrm{NH}_{3}$ PET, it seems advisable to use at least 6 iterations and 28 subsets. However, the optimal protocol may vary from vendor to vendor, and val- idation studies should ideally be performed before such substitution is done. Although aggressive smoothing may produce visually appealing images with reduced noise levels, it may cause an underestimation of absolute quantitative MBF. In selecting a reconstruction algorithm, an optimal balance between noise properties and diagnostic accuracy must be emphasized. CFR is a ratio and is not significantly affected by reconstruction algorithms as long as both rest and stress images are reconstructed the same way.

\section{ACKNOWLEDGMENT}

The authors acknowledge the excellent contributions of all nuclear technologists at the University of Washington. Without their effort, this work could not have been accomplished. This work was supported in part by NIH grant CA74135 and by the University of Washington Division of Cardiology.

\section{REFERENCES}

1. Schelbert HR, Phelps ME, Huang SC, et al. N-13 ammonia as an indicator of myocardial blood flow. Circulation. 1981;63:1259-1272.

2. Hutchins GD, Schwaiger M, Rosenspire KC, Krivokapich J, Schelbert H, Kuhl DE. Noninvasive quantification of regional blood flow in the human heart using $\mathrm{N}-13$ ammonia and dynamic positron emission tomographic imaging. J Am Coll Cardiol. 1990;15:1032-1042.

3. Muzik O, Beanlands RS, Hutchins GD, Mangner TJ, Nguyen N, Schwaiger M. Validation of nitrogen-13-ammonia tracer kinetic model for quantification of myocardial blood flow using PET. J Nucl Med. 1993;34:83-91.

4. Nitzsche EU, Choi Y, Czernin J, Hoh CK, Huang SC, Schelbert HR. Noninvasive quantification of myocardial blood flow in humans: a direct comparison of the $\left[{ }^{13} \mathrm{~N}\right]$ ammonia and the $\left[{ }^{15} \mathrm{O}\right]$ water techniques. Circulation. 1996;93:2000-2006.

5. Kotzerke J, Glatting G, van den Hoff J, et al. Validation of myocardial blood flow estimation with nitrogen-13 ammonia PET by the argon inert gas technique in humans. Eur J Nucl Med. 2001;28:340-345.

6. Hudson HM, Larkin RS. Accelerated image reconstruction using ordered subsets of projection data. IEEE Trans Med Imaging. 1994;13:601-609. 
7. Bacharach SL, Bax JJ, Case J, et al. PET myocardial glucose metabolism and perfusion imaging. Part 1. Guidelines for data acquisition and patient preparation. J Nucl Cardiol. 2003;10:543-556.

8. Razifar P, Sandstrom M, Schnieder H, et al. Noise correlation in PET, CT, SPECT and PET/CT data evaluated using autocorrelation function: a phantom study on data, reconstructed using FBP and OSEM [electronic resource]. BMC Med Imaging. Aug 25, 2005;5:5. Available at: http://www.biomedcentral.com/ 1471-2342-5-5. Accessed June 7, 2007.

9. Boellaard R, van Lingen A, Lammertsma AA. Experimental and clinical evaluation of iterative reconstruction (OSEM) in dynamic PET: quantitative characteristics and effects on kinetic modeling. J Nucl Med. 2001;42:808-817.

10. Wang C, Snyder W, Bilbro G, Santago P. Performance evaluation of filtered backprojection reconstruction and iterative reconstruction methods for PET images. Comput Biol Med. 1998;28:13-25.
11. Lubberink M, Boellaard R, van der Weerdt AP, Visser FC, Lammertsma AA. Quantitative comparison of analytic and iterative reconstruction methods in 2- and 3-dimensional dynamic cardiac ${ }^{18}$ F-FDG PET. J Nucl Med. 2004;45:2008-2015.

12. Link JM, Stratton JR, Levy WC, et al. PET measures of pre- and post-synaptic cardiac beta adrenergic function. Nucl Med Biol. 2003;30:795-803.

13. Khorsand A, Graf S, Pirich C, et al. Assessment of myocardial perfusion by dynamic N-13 ammonia PET imaging: comparison of 2 tracer kinetic models. J Nucl Cardiol. 2005;12:410-417.

14. Beanlands RS, Muzik O, Melon P, et al. Noninvasive quantification of regional myocardial flow reserve in patients with coronary atherosclerosis using nitrogen13 ammonia positron emission tomography: determination of extent of altered vascular reactivity. J Am Coll Cardiol. 1995;26:1465-1475.

15. Heymann MA, Payne BD, Hoffman JIE, Rudolph AM. Blood flow measurements with radionuclide-labeled particles. Prog Cardiovasc Dis. 1977;20:55-79. 